\title{
Changes of kinematic parameters of lower extremities with gait speed: a 3D motion analysis study
}

\author{
Jung Won Kwon, PhD, $\mathrm{PT}^{1)}$, Sung Min Son, PhD, $\mathrm{PT}^{2)}$, Na Kyung Lee, PhD, $\mathrm{PT}^{1)^{*}}$ \\ 1) Department of Physical Therapy, College of Rehabilitation Science, Daegu University: 5 Jilyang, \\ Gyeongsan-si, Kyeongbuk 712-714, Republic of Korea \\ 2) Department of Physical Therapy, College of Health Science, Cheongju University, Republic of Korea
}

\begin{abstract}
Purpose] The purpose of this study was to investigate the changes in hip, knee and ankle kinematic variables of the lower extremities at different gait speeds. [Subjects and Methods] Forty healthy subjects who had no previous history of neurological, musculo-skeletal or other medical conditions that could affect gait were recruited. The subjects were asked to walk $10 \mathrm{~m}$ down a walkway at three different gait speeds: normal gait speed, and selfselected fast, and slow speeds. The experimental order was randomly chosen across these gaits. The hip, knee and ankle kinematic data were evaluated using a VICON 3D motion analysis system and force plates. [Results] The flexion peak and external rotation peak of the knee joint significantly increased with the increase of gait speed. The plantarflexion peaks of the ankle joint significantly increased with increase of gait speed. However, none of the kinematic data of the hip joint were significantly dependent on increase of gait speed. [Conclusion] The relationship of the knee and ankle joint can be described as coupling motion which is dependent on gait speed. Our present findings suggest that coupling motion of the knee joint and plantarflexion of the ankle joint significantly increase with increase of gait speed. These results will provide important insight into gait mechanisms for the evaluation of pathological populations.

Key words: Gait speed, 3D motion analysis, Lower extremity
\end{abstract}

(This article was submitted Jul. 28, 2014, and was accepted Sep. 2, 2014)

\section{INTRODUCTION}

Human gait is important for efficient and safe locomotion $^{1)}$. Gait analysis provides us with important information on the functional capacity of patients when making disability assessments, and in intervention and treatment planning 2,3 ). The gait speed substantially influences the gait pattern, and kinematic and kinetic characteristics ${ }^{4,5)}$.

When quantifying human gait by kinematic and kinetic characteristics, subjects are typically asked to walk at what is called their normal gait speed ${ }^{6,7)}$. In general, normal gait speeds have been shown to range from 1.05 to $1.43 \mathrm{~m} / \mathrm{s}$ in healthy adults ${ }^{8,9)}$. These subject-specific speeds of healthy subjects have been used to produce normative gait data that can be used as a reference when abnormal and/or pathological gait is assessed. A possible limitation of this procedure is that slower gait speeds may be associated with decreased joint movements and joint kinetics. At faster gait speeds, clinical evidence suggests that increased joint power is

*Corresponding author. Na Kyung Lee (E-mail: dlskrud21@ hanmail.net)

C2015 The Society of Physical Therapy Science. Published by IPEC Inc. This is an open-access article distributed under the terms of the Creative Commons Attribution Non-Commercial No Derivatives (by-ncnd) License $<$ http://creativecommons.org/licenses/by-nc-nd/3.0/>. responsible for certain types of overuse injuries of the musculoskeletal system $^{10,11)}$. Thus, it is generally accepted that normal gait parameters follow a consistent pattern of change in response to changes in gait speed.

Many previous studies have investigated the relationship between kinematics of the lower limbs and gait speed ${ }^{7,12,13)}$. In addition, gait speed has been shown to have a significant effect on gait kinematics and clinicians can provide normative kinematic data as goals for patients who walk slowly at their normal gait speed. Recent research has proposed the use of self-selected gait speeds to specify walking speed, because it offers the advantages of allowing comparisons between speeds, while concurrently allowing the subjects to act as their own control to normalize gait parameters ${ }^{10,11)}$. Thus, self-selected gait speeds are recognized as valid and sensitive measures of gait performance, and identifying kinematic profiles that relate to gait speed could provide guidance for intervention strategies aimed at improving normal gait. Recent studies have addressed gait movement patterns using three-dimensional (3D) motion analysis systems, but these have been limited to the sagittal plane and have neglected the frontal and transverse planes ${ }^{7,}{ }^{14)}$. Both the frontal and transverse planes are important because gait performance occurs in three-dimensional space. Therefore, we investigated the individual kinematic gait profiles of normal subjects in all three planes and identified frontal and transverse profiles in addition to the sagittal profile at self- 
Table 1. Comparison of Kinematic from gait speed on lower extremity joint $\left({ }^{\circ}\right)$

\begin{tabular}{|c|c|c|c|c|c|}
\hline Joint & Plane & Angle & SS & NS & FS \\
\hline \multirow{6}{*}{ Hip joint } & \multirow{2}{*}{ Sagittal } & Flexion peak & $26.3 \pm 6.05$ & $28.6 \pm 5.20$ & $30.3 \pm 7.40$ \\
\hline & & Extension peak & $10.3 \pm 5.67$ & $12.9 \pm 5.82$ & $13.8 \pm 6.04$ \\
\hline & \multirow{2}{*}{ Frontal } & Abduction peak & $2.78 \pm 5.09$ & $4.15 \pm 4.05$ & $4.62 \pm 4.20$ \\
\hline & & Adduction peak & $5.69 \pm 8.02$ & $8.62 \pm 3.21$ & $7.66 \pm 8.56$ \\
\hline & \multirow{2}{*}{ Trans } & External rotation peak & $21.9 \pm 12.2$ & $16.0 \pm 12.4$ & $16.3 \pm 14.3$ \\
\hline & & Internal rotation peak & $-1.07 \pm 9.53$ & $8.20 \pm 11.3$ & $9.41 \pm 11.9$ \\
\hline \multirow{6}{*}{ Knee joint } & \multirow{2}{*}{ Sagittal } & Flexion peak & $44.3 \pm 6.61$ & $54.3 \pm 6.83$ & $53.3 \pm 6.03$ \\
\hline & & Extension peak & $1.49 \pm 5.22$ & $1.90 \pm 4.56$ & $2.26 \pm 5.79$ \\
\hline & \multirow{2}{*}{ Frontal } & Abduction peak & $11.4 \pm 16.9$ & $15.8 \pm 10.7$ & $11.1 \pm 4.16$ \\
\hline & & Adduction peak & $-0.05 \pm 7.80$ & $-2.35 \pm 4.45$ & $-1.08 \pm 8.80$ \\
\hline & \multirow{2}{*}{ Trans } & External rotation peak ${ }^{\dagger *}$ & $5.51 \pm 4.01$ & $7.70 \pm 5.74$ & $8.07 \pm 4.31$ \\
\hline & & Internal rotation peak & $3.39 \pm 12.1$ & $3.58 \pm 8.58$ & $7.70 \pm 8.73$ \\
\hline \multirow{6}{*}{ Ankle joint } & \multirow{2}{*}{ Sagittal } & Dorsiflexion peak & $11.8 \pm 5.21$ & $12.7 \pm 3.26$ & $10.3 \pm 8.87$ \\
\hline & & Plantarflexion peak ${ }^{\dagger}$ & $7.85 \pm 7.82$ & $12.9 \pm 9.11$ & $16.1 \pm 6.83$ \\
\hline & \multirow{2}{*}{ Frontal } & Abduction peak & $4.53 \pm 4.33$ & $4.95 \pm 2.77$ & $4.90 \pm 2.98$ \\
\hline & & Adduction peak & $-0.42 \pm 3.53$ & $-0.90 \pm 1.99$ & $-1.36 \pm 2.37$ \\
\hline & \multirow{2}{*}{ Trans } & External rotation peak & $-4.40 \pm 11.7$ & $-4.59 \pm 12.4$ & $-3.65 \pm 9.84$ \\
\hline & & Internal rotation peak & $23.9 \pm 10.2$ & $25.6 \pm 10.2$ & $25.2 \pm 10.9$ \\
\hline
\end{tabular}

'Significant difference between the SS and FS groups $(\mathrm{p}<0.05)$.

${ }^{\dagger}$ Significant difference between the SS and NS groups $(\mathrm{p}<0.05)$.

selected gait speeds.

\section{SUBJECTS AND METHODS}

Forty healthy subjects who had no previous history of neurological, musculo-skeletal or other medical conditions that could have affected gait were recruited. All the subjects understood the purpose of this study and gave their written and informed consent prior to their participation. The study protocol was approved by the local Institutional Review Board and complied with the ethical principles of the Declaration of Helsinki.

The subjects were first asked to walk along a $10 \mathrm{~m}$ walkway at a comfortable pace. The procedure was repeated at two different gait speeds, performed in a random order, to give three sequences of data for each subject. The three gait speeds were as follows and were always performed at a selfselected speed: slow speed (SS): a gait somewhat slower than normal walking speed; normal speed (NS): a gait at a normal, comfortable speed; fast speed (FS): a gait somewhat faster than normal walking speed ${ }^{13)}$. The experimental order of the self-selected gait speeds.

A twelve-camera video-based motion analysis system (Vicon, UK) was used to record joint angles. Sixteen reflecting markers were placed on specific anatomical landmarks on the hip (ASISs and PSISs) as well as on each mid-thigh (the lower lateral $1 / 3$ of the thigh), knee (lateral epicondyle), mid-tibia (lower $1 / 3$ of the shank and lateral malleolus), and foot (second metatarsal head and heel) for capturing joint motion. The marker protocol was based on the Plug-in-gait marker set. The motion capture rate was 120 fps. Two force plates (Advanced Mechanical Technology Inc., USA) were placed at the centre of the $10 \mathrm{~m}$ walkway to measure the gait cycle. Data collected from the force plates were digitized at $500 \mathrm{~Hz}$ and low-pass filtered at $1,000 \mathrm{~Hz}$. The system was capable of measuring separate repeated steps over successive strides for extended trials. Only trials that included three consecutive clean force platform data and complete data were considered.

The statistical analysis was performed using SPSS ver. 12.0. Demographic data such as age, height and weight were analyzed by one-way ANOVA. The values of the different gait speeds were compared using one-way ANOVA and least-squares difference as a post hoc test. Statistical significance was accepted for values of $\mathrm{p}<0.05$.

\section{RESULTS}

The subjects had an average age of $23.16 \pm 3.76$ years, an average body mass of $65.88 \pm 6.97 \mathrm{~kg}$, and an average height of $171.04 \pm 3.52 \mathrm{~cm}$.

The flexion peak and external rotation peak of the knee joint significantly increased with increase of gait speed $(p<0.05)$. The results of the LSD posthoc test show that the flexion peak of the knee joint was significantly different between SS and NS $(p<0.05)$ (Table 1). The external rotation peak of the knee joint was significantly different between SS and NS, SS and FS $(\mathrm{p}<0.05)$ (Table 1). Plantarflexion peaks of the ankle joint significantly increased with increase of gait speed $(p<0.05)$. The results of the LSD posthoc test show that plantarflexion of the ankle joint was significantly different between SS and NS, and SS and FS $(p<0.05)$ (Table 1).

\section{DISCUSSION}

In the current study, we found that between the pre-swing 
and the mid-swing the flexion peak and the external rotation peak of the knee joint and the plantarflexion peak of the ankle joint significantly increased with the increase of the gait speed. These results imply that there is a need for greater power generation at higher gait speeds for propelling the body forward, and it is accompanied by coupling motion of the external rotation of the knee joint.

Gait speed has an important influence on the kinematic parameters of the lower extremity joints. In particular, the kinematic parameters of the knee and ankle joints have a positive relationship with gait speed ${ }^{13,15,16)}$. In this study, the knee flexion peak during pre-swing and mid-swing significantly increased with the increase of gait speed. The knee flexion angle in the gait cycle has a significant positive correlation with the gait speed, according to previous studies $^{13,16)}$, indicating that the angle of the knee flexion increases with increase of gait speed. It is possible that this is due to the need for greater shock absorption at higher speeds. In addition, our findings show that the external rotation peak of the knee joint significantly increased with increase of gait speed. Previous studies have reported that one joint segment of the lower extremities affects the others during the gait. These relationships are described as coupling motion ${ }^{16,17)}$. Therefore, we think that gait speed is affected by coupling motion of the lower extremity. Our present findings support the findings of other studies of gait mechanism. The plantarflexion peak of the ankle joint significantly increased with increase of gait speed. This result is in agreement with other studies that have suggested that plantarflexion of the ankle joint is closely related to gait speed. In addition, the ankle joint plays an important role in changing gait speed, and it has been suggested that plantarflexion is a strong predictor of step length and gait speed ${ }^{18-20)}$.

In conclusion, we examined the changes in the lower extremity joints at different gait speeds using a 3-dimensional motion analysis system. Our results suggest that coupling motion of the knee joint and plantarflexion of the ankle joint significantly increase with increase of the gait speed. These results provide important insights into the gait mechanisms underlying speed regulation in walking and provide a foundation on which to investigate the influence of walking speed on various measures of interest in pathological populations. In this study, we restricted recruitment to males of standard height and weight to negate the effects of external factor. Therefore, further studies involving a larger population are suggested.

\section{REFERENCES}

1) Han JT, Kwon YH, Park JW, et al.: Three-dimension kinematic analysis during upslope walking with different inclinations by healthy adults. J PhysTherSci, 2009, 21: 385-391. [CrossRef]

2) Kim TY, Yoo WG, An DH, et al.: The effects of different gait speeds and lower arm weight on the activities of the latissimusdorsi, gluteus medius, and gluteus maximus muscles. J PhysTherSci, 2013, 25: 1483-1484. [Medline] [CrossRef]

3) Jordan K, Challis JH, Newell KM: Walking speed influences on gait cycle variability. Gait Posture, 2007, 26: 128-134. [Medline] [CrossRef]

4) Benedetti MG, Catani F, Leardini A, et al.: Data management in gait analysis for clinical applications. ClinBiomech (Bristol, Avon), 1998, 13: 204-215. [Medline] [CrossRef]

5) Robinson JL, Smidt GL: Quantitative gait evaluation in the clinic. Phys Ther, 1981, 61: 351-353. [Medline]

6) Eppeland SG, Myklebust G, Hodt-Billington C, et al.: Gait patterns in subjects with rheumatoid arthritis cannot be explained by reduced speed alone. Gait Posture, 2009, 29: 499-503. [Medline] [CrossRef]

7) Latt MD, Menz HB, Fung VS, et al.: Walking speed, cadence and step length are selected to optimize the stability of head and pelvis accelerations. Exp Brain Res, 2008, 184: 201-209. [Medline] [CrossRef]

8) Al-Obaidi S, Wall JC, Al-Yaqoub A, et al.: Basic gait parameters: a comparison of reference data for normal subjects 20 to 29 years of age from Kuwait and Scandinavia. J Rehabil Res Dev, 2003, 40: 361-366. [Medline] [CrossRef]

9) Oberg T, Karsznia A, Oberg K: Basic gait parameters: reference data for normal subjects, 10-79 years of age. J Rehabil Res Dev, 1993, 30: 210-223. [Medline]

10) Mochon S, McMahon TA: Ballistic walking. J Biomech, 1980, 13: 49-57. [Medline] [CrossRef]

11) Richards C, Malouin F, Dumas F, et al.: Gait velocity as an outcome measure of locomotor recovery agrer stroke. In: Craik R, Oatic C, editors. Gait analysis: theory and applications. St. Louis: Mosby, 1995, pp 355-64.

12) Kang HG, Dingwell JB: Separating the effects of age and walking speed on gait variability. Gait Posture, 2008, 27: 572-577. [Medline] [CrossRef]

13) Lelas JL, Merriman GJ, Riley PO, et al.: Predicting peak kinematic and kinetic parameters from gait speed. Gait Posture, 2003, 17: 106-112. [Medline] [CrossRef]

14) Jagadamma KC, Owen E, Coutts FJ, et al.: The effects of tuning an anklefoot orthosis footwear combination on kinematics and kinetics of the knee joint of an adult with hemiplegia. Prosthet Orthot Int, 2010, 34: 270-276. [Medline] [CrossRef]

15) Corfr'e L, Lythgo N, Morqan D, et al.: Aging modifies joint power and work when gait speeds are matched. Gait Posture, 2011, 33: 484-489. [Medline] [CrossRef]

16) Hanlon M, Anderson R: Prediction methods to account for the effect of gait speed on lower limb angular kinematics. Gait Posture, 2006, 24: 280287. [Medline] [CrossRef]

17) Bellchamber TL, van den Bogert AJ: Contributions of proximal and distal moments to axial tibial rotation during walking and running. J Biomech, 2000, 33: 1397-1403. [Medline] [CrossRef]

18) Hoch MC, McKeon PO, Andreatta RD: Plantar vibrotactile detection deficits in adults with chronic ankle instability. Med Sci Sports Exerc, 2012, 44: 666-672. [Medline] [CrossRef]

19) McGibbon CA: Toward a better understanding of gait changes with age and disablement: neuromuscular adaptation. Exerc Sport Sci Rev, 2003, 31: 102-108. [Medline] [CrossRef]

20) Monaco V, Rinaldi LA, Macrì G, et al.: During walking elders increase efforts at proximal joints and keep low kinetics at the ankle. ClinBiomech (Bristol, Avon), 2009, 24: 493-498. [Medline] [CrossRef] 